\title{
Evaluation of a Bubble CPAP System for Low Resource Settings
}

\author{
Michelle L Dundek, Ellie K Ng, Abigail M Brazil, Robert M DiBlasi, Jonathan A Poli, and \\ Thomas F Burke
}

\begin{abstract}
BACKGROUND: Despite its established safety, efficacy, and relative simplicity, CPAP treatment is not widely available for newborns and infants in low- and middle-income settings. A novel bubble CPAP system was designed to address the gaps in quality and accessibility of existing CPAP systems by providing blended, humidified, and pressurized gases without the need for electricity, compressed air, or manual power. This was the first study that tested the performance of the system with a simulated patient model. METHODS: In a spontaneously breathing 3dimensional printed nasal airway model of a preterm neonate, CPAP performance was assessed based on delivered pressure, oxygen level, and humidity at different settings. RESULTS: Preliminary device performance characteristics were within 5\% among 3 separate devices. Performance testing showed accurate control of CPAP and oxygen concentration at all settings with the bubble CPAP system. Lung model pressure and oxygen concentration were shown to stay within $\pm 0.5 \mathrm{~cm} \mathrm{H}_{2} \mathrm{O}$ and $\pm 4 \%$ of full scale of the device settings, respectively, with relative humidity $>80 \%$. CONCLUSIONS: Performance testing of the bubble CPAP system demonstrated accurate control of CPAP and oxygen concentration with humidity levels suitable for premature newborns on noninvasive support. Key words: Bubble CPAP; global health; respiratory distress syndrome; humidification. [Respir Care 2021;66(10):1572-1581. (C) 2021 Daedalus Enterprises]
\end{abstract}

\section{Introduction}

Nearly 4 million infants die throughout the world each year, with 1 million dying principally from respiratory insufficiency in low- and middle-income countries that lack respiratory support devices and technologies commonly used in high resource settings. ${ }^{1-5}$ The absent technologies include advanced invasive mechanical ventilators, but they also include relatively simple interventions, for example,

\footnotetext{
Ms Dundek, Ms Ng, Ms Brazil, and Dr Burke are affiliated with the Vayu Global Health Foundation, Boston, Massachusetts. Ms Dundek and Dr Burke are affiliated with the Global Health Innovation Laboratory, Department of Emergency Medicine, Massachusetts General Hospital, Boston, Massachusetts. Mr DiBlasi and Mr Poli are affiliated with the Department of Respiratory Care Therapy, Seattle Children's Hospital and Regional Medical Center, Seattle, Washington. Mr DiBlasi is affiliated with the Center for Integrative Brain Research, Seattle Children's Research Institute, Seattle, Washington. Dr Burke is affiliated with the Harvard Medical School, Boston, Massachusetts. Dr Burke is affiliated with the Harvard T.H. Chan School of Public Health, Boston, Massachusetts.
}

Supplementary material related to this paper is available at http://www. rcjournal.com. noninvasive ventilation. Nasal CPAP is a form of non-invasive respiratory support that has been used for almost 50 years in high resource settings. It is associated with lower indicators of lung injury and inflammation, pulmonary growth arrest, and chronic lung disease than invasive mechanical ventilators. ${ }^{6-11}$ When it is made available in lowand middle-income countries, nasal CPAP has been shown to reduce mortality in preterm neonates by $66 \% .^{12}$ As such, the World Health Organization (WHO) strongly recommends CPAP for newborns diagnosed with respiratory distress syndrome. ${ }^{13}$ The American Academy of Pediatrics

\footnotetext{
The Vayu Global Health Foundation provided the bubble CPAP systems for this study.

Mr DiBlasi discloses relationships with Draeger Medical, Bunnell Medical, Vapotherm, and Vero Biotech. Ms Dundek and Ms $\mathrm{Ng}$ are engineers with Vayu Global Health Innovations. The other authors have disclosed no conflicts of interest.

Correspondence: Michelle L Dundek, Vayu Global Health Foundation, 22 Welgate Road, Medford, MA 02155.E-mail: michelledundek@gmail.com.
}

DOI: $10.4187 /$ respcare. 08948 
also recommends that nasal CPAP and other oxygen blending systems are made available for all preterm births ${ }^{14}$; and others have pointed out the potential of nasal CPAP to support patients in transit. ${ }^{5-17}$

Despite its established safety, efficacy, and relative simplicity, nasal CPAP treatment is not widely available in low- and middle-income countries. In India, for example, a 2020 survey found that only one third of district hospital neonatal units have any access to CPAP. ${ }^{18}$ There are many barriers to accessing nasal CPAP, including cost; the need for electricity; the need for compressed medical air and blending systems; and availability of skilled human resources for training, maintenance, and patient monitoring. As a result, care providers in low- and middle-income settings often resort to improvising nasal bubble CPAP (bubble CPAP) systems; approximately one third of the hospitals in the Indian survey ${ }^{18}$ using nasal CPAP used home-made bubble CPAP systems; these systems were made from supplies on hand, which typically include a compressed and nonhumidified pure oxygen $(100 \%)$ gas source. They often give poor quality CPAP because the thin nasal cannulas that they use are difficult to exhale through ${ }^{19,20}$ and they lead to prolonged exposure to pure oxygen, which can cause damage to the eyes, lungs, and brain.

Several low-cost bubble CPAP systems have been developed in recent years to address the demand for affordable methods of delivering CPAP. ${ }^{21-27}$ These devices have some significant advantages over previous devices, including successfully eliminating the need for compressed air. However, these more affordable bubble CPAP systems still face formidable barriers to adoption in many facilities. They all require continuous electricity to humidify gases or to blend air and oxygen, but a 2014 WHO Surgical Assessment Tool survey ${ }^{28}$ of $\sim 800$ lowincome hospitals found that only 59\% of health-care facilities have reliable electricity access. In addition, many low-cost CPAP systems do not provide the same quality of CPAP as established, expensive devices. Lowcost CPAP systems often do not include humidification, have large amounts of dead space that cause the patient to rebreathe carbon dioxide and use high-resistance circuits that have a large imposed work of breathing. ${ }^{19}$ Therefore, there remains an unmet need for a system that provides high-quality bubble CPAP, does not require electricity or compressed air, is easy to use, and is low cost.

Mollazadeh-Moghaddam et $\mathrm{al}^{29}$ described a low-cost method to blend ambient air and pressurized oxygen without electricity by using a unique fixed performance Venturi blender. An adjustable version of this Venturi blender was created and combined with a humidifier, low-resistance breathing circuit, and water column to create a novel bubble CPAP system (Vayu Global Health Innovations, Boston, Massachusetts). The following descriptive studies in vitro were designed to evaluate the performance of the novel

\section{QUICK LOOK}

\section{Current knowledge}

CPAP has been used for decades to treat newborns and infants with respiratory distress in high resource settings. Results of studies have shown that CPAP can reduce mortality when implemented in low resource settings, but CPAP treatment remains unavailable in many low-resource hospitals due to its cost and other barriers. Several low-cost bubble CPAP systems have recently been designed to be more accessible, but these systems require continuous electricity and may be expensive.

\section{What this paper contributes to our knowledge}

A newborn and infant CPAP system has been developed that does not require electricity or compressed air. This study evaluated device performance in a lung model. The device provided pressure, oxygen concentrations, and humidification to an appropriate range and accuracy for newborns and infants.

bubble CPAP system based on clinically relevant parameters critical to effective CPAP therapy by using a realistic, spontaneously breathing patient model.

\section{Methods}

We designed and conducted studies in vitro in 3 separate stages. The first stage evaluated pressure, oxygen concentration delivery, and humidity by using a specialized temperature and humidity chamber at all device settings for 3 samples of the bubble CPAP system. These studies evaluated interdevice variability and performance stability and can be found by accessing the online supplement (see the supplementary materials at http://www.rcjournal.com). After this testing, the second stage evaluated delivered pressure and oxygen concentration in a high-fidelity realistic breathing lung model by using appropriate flow settings for low birthweight newborns, who represent the most common patient population that requires CPAP for respiratory distress syndrome and surfactant deficiency. The third stage evaluated relative humidity $(\mathrm{RH})$ levels within a nonheated lung model by using a bellows, a 3-dimensional model, and a hygrometer. The laboratory setting was maintained at low ambient humidity to evaluate humidity output without rebreathing of exhaled heated humidity.

\section{The Bubble CPAP System}

The bubble CPAP system delivers humidified, filtered, pressurized, and oxygen-enriched air to the patient (Fig. 1A) 


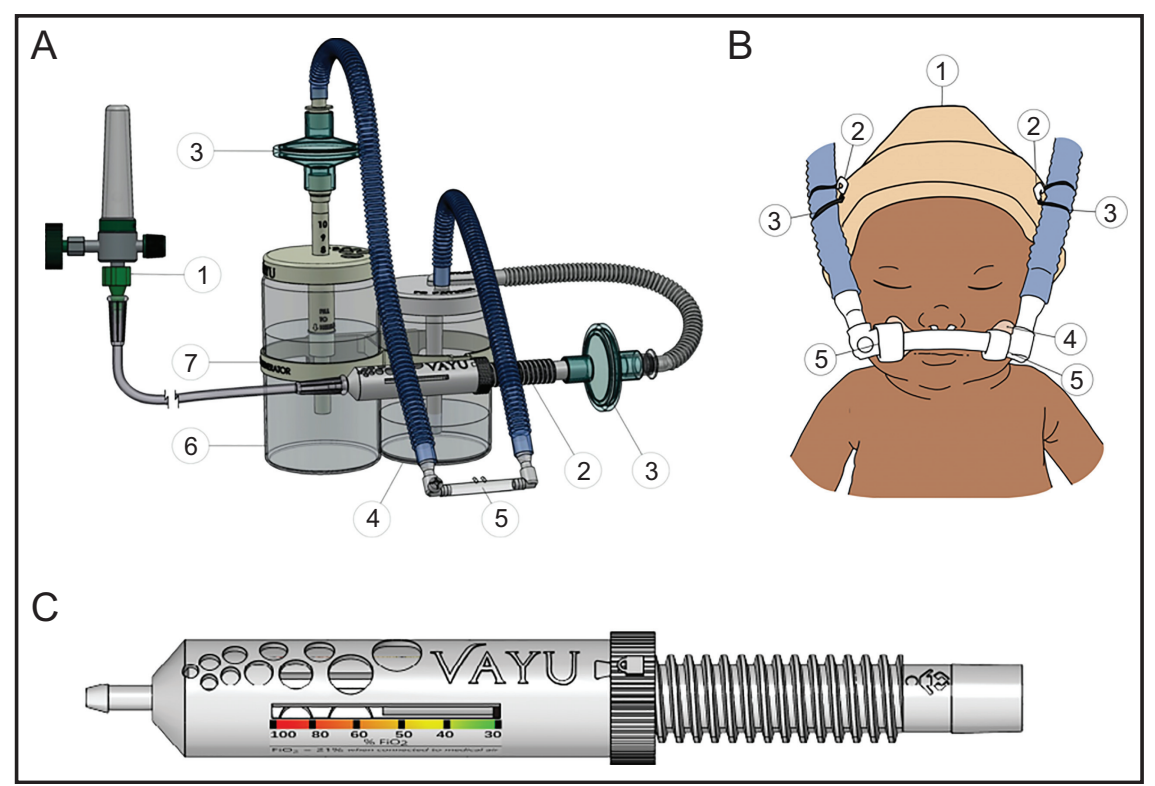

Fig. 1. A: The bubble CPAP system composed of (1) an external pressurized oxygen source; (2) Venturi blender; (3) 2 bacterial and viral filters, one on the inspiratory limb and one on the expiratory limb; (4) a humidifier; (5) nasal prongs; (6) a water column with an adjustable wand to control the delivered pressure from 4 to $10 \mathrm{~cm} \mathrm{H}_{2} \mathrm{O}$; and (7) warmer bracket that stabilizes the system and allows it to be placed inside infant radiant warmers. B: The patient interface, composed of (1) hat; (2) 2 safety pins, (3) 2 rubber bands, (4) hook tape moustache, and (5) loop tape strips. C: The Venturi blender is the distinguishing feature of the bubble CPAP system; it delivers $30-100 \%$ oxygen by mixing pressurized oxygen and ambient air.

via secured nasal prongs (Fig. 1B). The system requires a 50-psi source of medical-grade oxygen with an adjustable flow meter. Oxygen enters the circuit and passes through an adjustable Venturi blender (Fig. 1C), where it mixes with ambient air to create a source of oxygen-enriched air of adjustable concentration. The mixture is filtered, humidified, and delivered to the patient through short bi-nasal prongs. Exhaled gases exit the system by bubbling out through the water column, which generates CPAP. The amount of pressure delivered to the patient is determined by the depth of submersion of the expiratory tubing in the water column (4-10 $\mathrm{cm} \mathrm{H}_{2} \mathrm{O}$ ). The volumetric flow of the source gas is set to the minimum flow that maintains continuous bubbling throughout the respiratory cycle at the set CPAP level. ${ }^{30}$

The bubble CPAP system Venturi blender, an adjustable concentration Venturi blender that can handle operational low flows and relatively thin tubing found in neonatal circuits, ${ }^{29}$ generates oxygen-enriched air by augmenting the flow of pure oxygen from a cylinder with entrained ambient air. It can be adjusted from 30 to $100 \%$ oxygen by turning the threaded portion to position an indicator along a numbered scale. A nut can be tightened to lock the position, and a swivel connector downstream allows the Venturi blender to be adjusted during treatment without coiling the tubing. A bacterial viral filter (Product number: VF-2160, Great Group Medical, Changhua, Taiwan) is positioned downstream of the Venturi blender and upstream of the humidifier. The bubble-through humidifier comprises a custom molded lid screwed onto a plastic reservoir and operates passively as the enriched, filtered gas passes through a small volume of water before exiting the chamber and entering the inspiratory limb of the breathing circuit. Standard 10-mm corrugated tubing connects the humidifier and water column to the nasal prongs (Infant Nasal Prongs, Great Group Medical). The expiratory limb of the breathing circuit is attached to a downstream filter, which connects to the wand of the custom-designed water column lid. The wand can be rotated to change the depth of submersion, which changes the amount of CPAP pressure delivered.

\section{Nasal Airway and Lung Models}

Nasal resistance in newborns accounts for nearly half of total airway resistance. ${ }^{31}$ As such, we designed a realistic replica of a nasal airway modeled from a computed tomography of an infant at 30 weeks of gestation (Fig. 2). This model has been described in detail elsewhere. ${ }^{32}$ The model did not have an oral airway opening; therefore, only closedmouth conditions were simulated. The nasal model was attached to 2 different spontaneously breathing preterm neonatal lung models for testing. Oxygen and pressure delivery was measured with the ASL 5000 Test Lung (Ingmar Medical, Pittsburgh, Pennsylvania), a digitally controlled, high-fidelity breathing simulator, which used a screw-drive controlled piston and mathematical modeling to simulate size- and disease-specific pulmonary mechanics (Fig. 3). Spontaneous breathing was simulated by using the preterm neonate normal values given in Table 1. The 

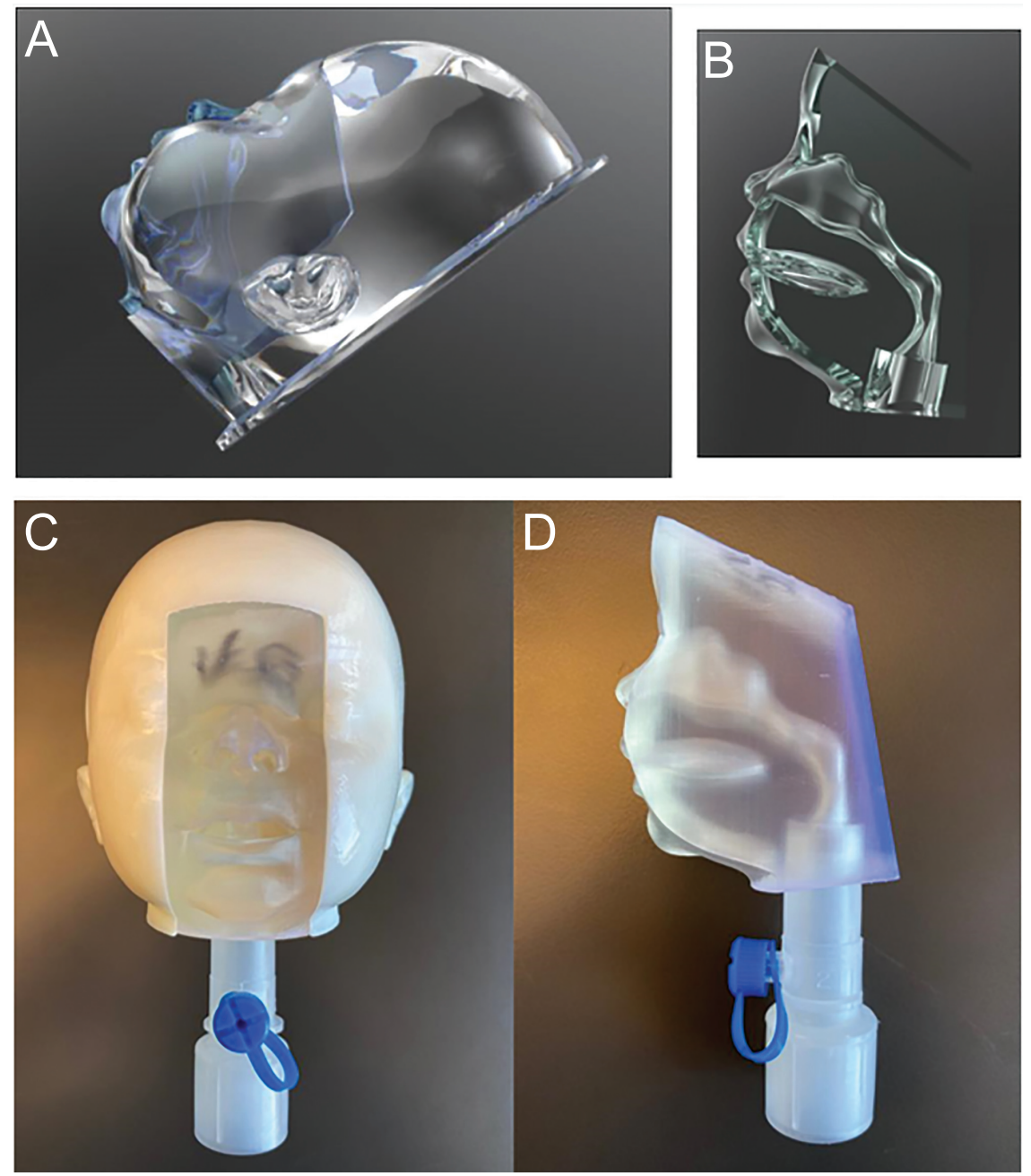

Fig. 2. Three-dimensional printed nasal airway of preterm infant taken from a 28 -wk-old subject. The full model is shown on the left (A) and the modular nasal airway is shown on the right (B). The nasal passages and closed oral cavity are clearly shown in B.

inspiratory-to-expiratory ratio was held constant at 1:3, and inspiratory effort was adjusted to maintain a constant tidal volume $(6 \mathrm{~mL} / \mathrm{kg})$. The second lung model, a Silastic infant test lung with compliance of $0.47 \mathrm{~mL} / \mathrm{cm} \mathrm{H}_{2} \mathrm{O}$ and resistance of $150 \mathrm{~cm} \mathrm{H}_{2} \mathrm{O} / \mathrm{L} / \mathrm{s}$ (model 191, Maquet, Wayne, New Jersey) (Fig. 4) was used to evaluate humidity levels.

\section{Lung Model Pressure and Oxygen Concentration Testing}

The bubble CPAP system, nasal model, and lung simulator were set up as shown in Figure 3. A pressurized oxygen gas cylinder connected to a regulator set at 50 psi (not shown) was attached to an adjustable flow meter. This flow meter was connected to the Venturi blender using oxygen tubing. A calibrated flow sensor (TSI INc Model \#5220A, Shoreview, Minnesota) was inserted between the Venturi blender and the upstream filter to monitor the total flow into the system (ie, blended air/oxygen flow), and direct readout of the flow was readily visible from an accompanying screen. To obtain the desired bias flow, the oxygen flow from the cylinder was titrated until the readout from the sensor matched. A flow of $6 \mathrm{~L} / \mathrm{min}$ was used because it was the minimum flow that maintained vigorous bubbling throughout the respiratory cycle at all CPAP levels.

The TSI flow meter was removed after measuring the flow and before testing. Nasal prongs (size 1) were inserted snugly into the nasal airway openings of the nasal model. Initially, the Venturi blender was set to $30 \%$ oxygen and the wand in the water column was set to $4 \mathrm{~cm} \mathrm{H}_{2} \mathrm{O}$. Once the readout of the oxygen concentration stabilized, 20 breathing cycles were recorded. The same steps were repeated for set CPAP levels of 6,8 , and $10 \mathrm{~cm} \mathrm{H}_{2} \mathrm{O}$, and the pressures were also applied at oxygen levels of 40,60, 80 , and $100 \%$ to record data for all setting combinations. Lung model pressures (end-expiratory pressure), oxygen 


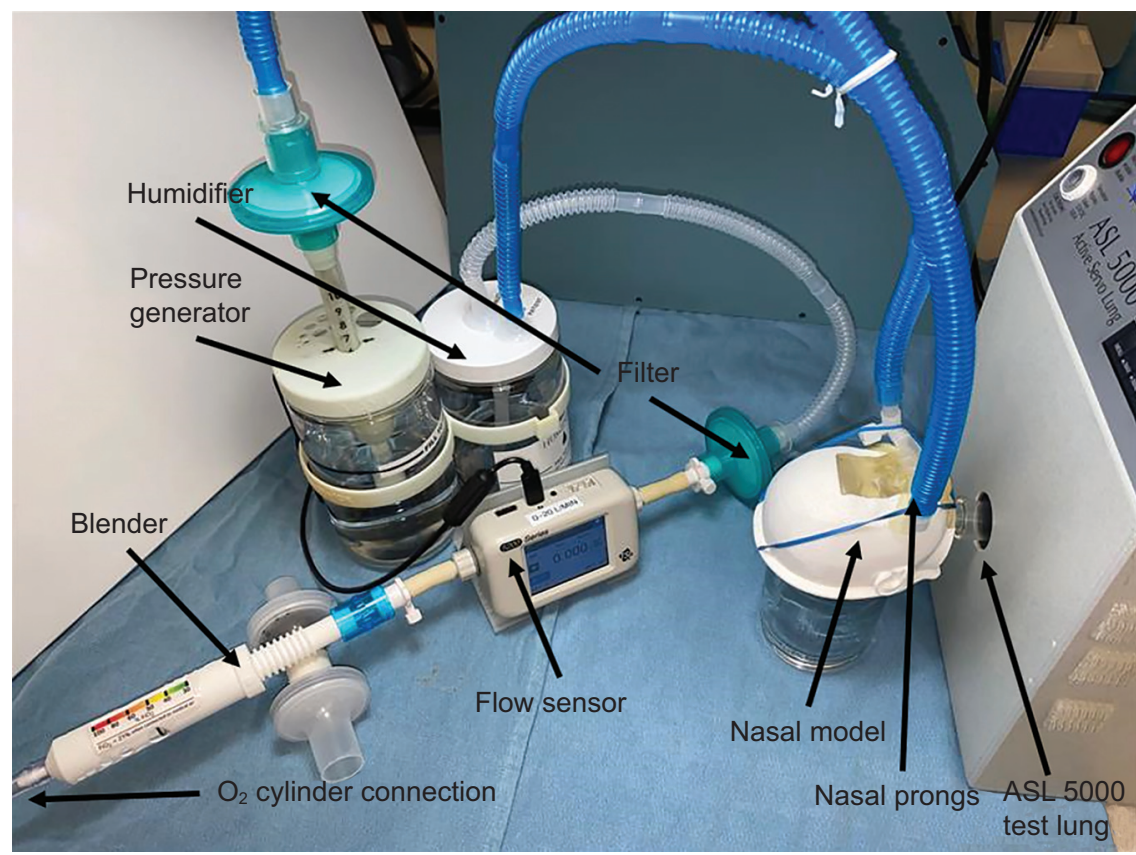

Fig. 3. Test setup for PEEP and oxygen concentration performance testing.

Table 1. Lung Model Configuration and Testing Conditions

\begin{tabular}{lc}
\hline \hline \multicolumn{1}{c}{ Variable } & Preterm Neonate, $1 \mathrm{~kg}$ \\
\hline Compliance, $\mathrm{mL} / \mathrm{cm} \mathrm{H}_{2} \mathrm{O}$ & 1 \\
Resistance, $\mathrm{cm} \mathrm{H} \mathrm{H}_{2} \mathrm{O} / \mathrm{L} / \mathrm{s}$ & 100 \\
Pleural pressure, $\mathrm{cm} \mathrm{H} \mathrm{O}_{2}$ & $7-8$ \\
Tidal volumes, $\mathrm{mL}$ & 6 \\
Frequency, breaths/min & 50 \\
Minute ventilation, $\mathrm{L} / \mathrm{min}$ & 0.3 \\
& \\
* Simulated pleural pressures were adjusted to maintain tidal volume of $6 \mathrm{~mL}$ at all testing \\
conditions.
\end{tabular}

concentration, and tidal volume were measured within the ASL 5000 Test Lung.

\section{Humidity Testing}

Humidification performance testing was conducted at a bias flow of $6 \mathrm{~L} / \mathrm{min}$ and a set CPAP of $5 \mathrm{~cm} \mathrm{H}_{2} \mathrm{O}$. The lung model was attached as shown in Figure 4. A hygrometer (Fisherbrand Traceable Temperature/Humidity Meter, Thermo Fisher Scientific, Waltham, Massachusetts) was inserted distal to the nasal model to measure the temperature and $\mathrm{RH}$ of the gases after they passed through the CPAP system and nasal passages. Breathing was simulated by manually generating breaths, distending and retracting the Silastic lung $\sim 2 \mathrm{~cm}$ in length. Two 1-way valves were placed in series to prevent rebreathing and recirculating of exhaled humidity from the lung model. For reference points, the RH of the laboratory ambient air and of the oxygen proximal to the cylinder were recorded and compared with the gas mixture exiting the bubble humidifier.

\section{Data Analysis}

The sampled data for each run of 20 breaths was extracted from the ASL 5000 Test Lung Software and saved to a spreadsheet (Excel, Microsoft, Redmond, Washington). The mean \pm SD was calculated for 20 simulated breaths by using Matlab (Mathworks, Natick, Massachusetts).

\section{Results}

Outcomes for delivered end-expiratory pressure (CPAP) and tidal volume based on different CPAP and oxygen concentration settings are shown in Figure 5. The tidal volume remained consistent across all combinations of CPAP and oxygen concentration settings, within $\pm 0.3 \mathrm{~mL}$ of the intended $6 \mathrm{~mL}$ volume. Flow remained consistent across all combinations of CPAP and oxygen concentration settings, with mean $\pm \mathrm{SD}$ registering $6.0 \pm 0.25 \mathrm{~L} / \mathrm{min}$. CPAP values registered within $\pm 0.5 \mathrm{~cm} \mathrm{H}_{2} \mathrm{O}$ of their set CPAP settings across all oxygen levels. Measured oxygen concentrations compared with the set percentage of oxygen are shown in Figure 6. Measured oxygen concentrations at settings of $30,40,60,80$, and $100 \%$ fell within $\pm 1, \pm 2, \pm 3, \pm 4$, and $\pm 2 \%$, respectively, of full scale of their intended setpoint value. Generally, higher CPAP levels generated 


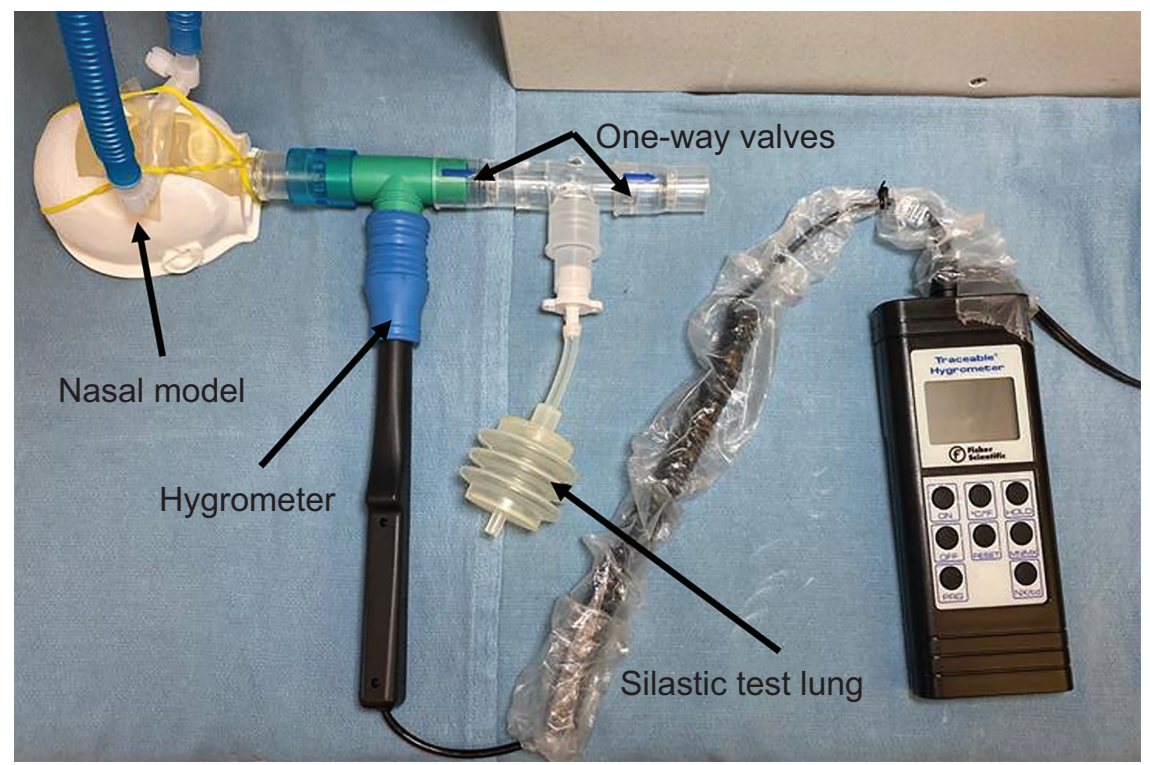

Fig. 4. Test setup for humidification performance testing.
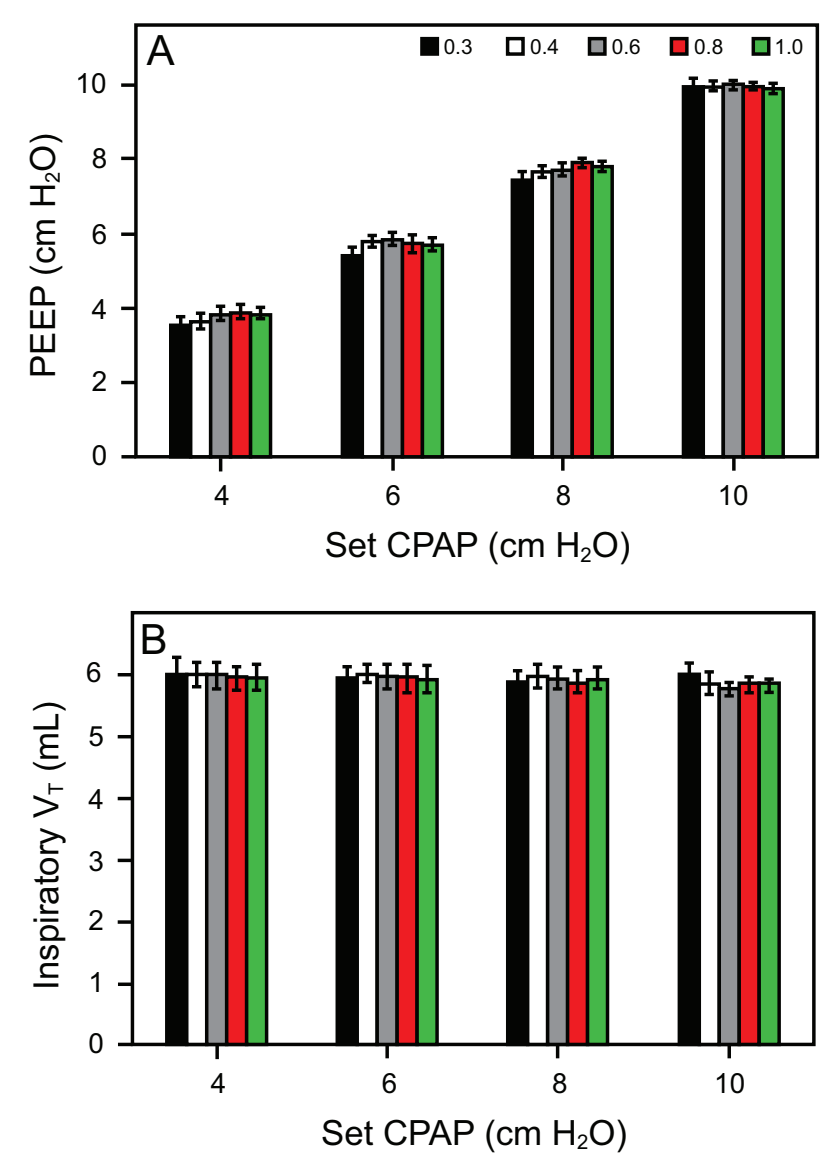

Fig. 5. Results for PEEP (A) and tidal volume (B) for all set CPAP levels and oxygen concentration settings. Each bar indicates the mean value of 20 breaths, and the error bars denote SD. slightly higher oxygen concentrations, particularly for the settings of $60 \%$ and $80 \%$.

Results from the humidity testing when using the nonheated valved lung model are shown in Figure 7. Reference values were observed to be $44.3 \pm 0.2 \% \mathrm{RH}$ for laboratory ambient air and $1.7 \pm 0.05 \% \mathrm{RH}$ proximal to the oxygen cylinder. There were only small differences in the RHs in the Silastic lung model that received humidified gas through the bubble humidifier at 100,60 , and $30 \%$ oxygen; at these settings, the RHs were $87.7 \pm 0.4 \%, 85.8 \pm 0.2 \%$, and $81.8 \pm 0.2 \%$, respectively. Combined effects from passive humidification and entrainment of ambient humidity, without assistance from heat, provided nearly 2 -fold greater RH than ambient and substantially greater humidity than the gas exiting the oxygen cylinder.

\section{Discussion}

The major findings from these studies in vitro with the bubble CPAP system showed accurate control of CPAP and oxygen concentration as well as adequate humidity delivery. Pressure and oxygen concentration were recorded well within $\pm 0.5 \mathrm{~cm} \mathrm{H}_{2} \mathrm{O}$ and $\pm 4 \%$, respectively, across all device settings. RHs $>80 \%$ were achieved without the aid of heat or moisture from actual nasal passageways. CPAP system performance is dependent on controlling pressure, blending oxygen, and humidifying breathing gases, and the bubble CPAP system delivered on all 3 criteria.

The bubble CPAP system maintains positive pressure by submerging the distal end of the breathing circuit into a 


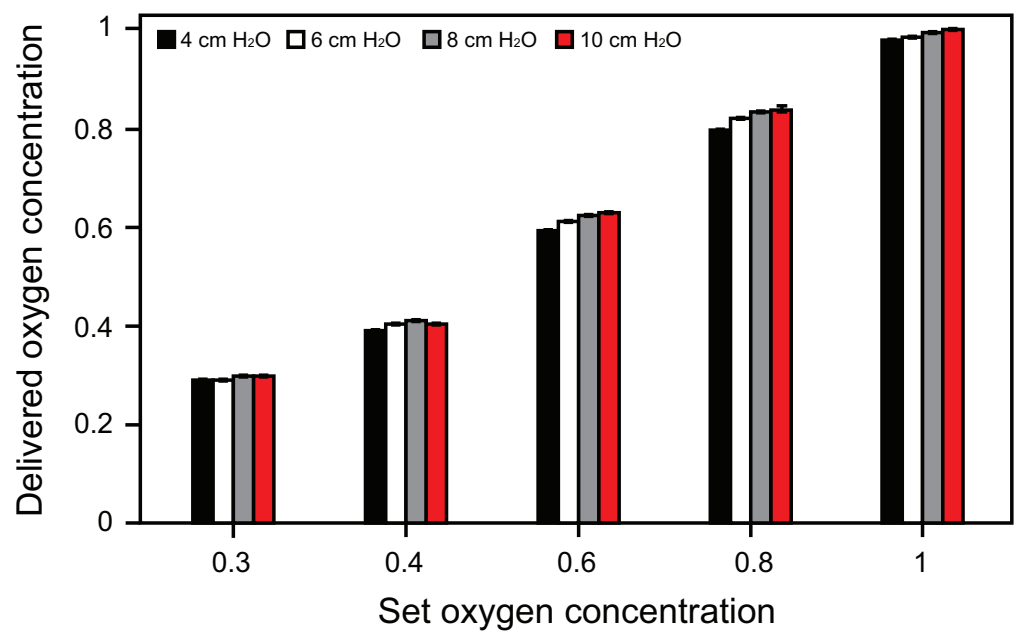

Fig. 6. Measured oxygen concentration as a function of a set oxygen concentration to illustrate the deviation from the intended value. Each bar indicates the mean value of 20 breaths and the error bars denote SDs. In each group, the different set CPAPs are denoted by different colors.

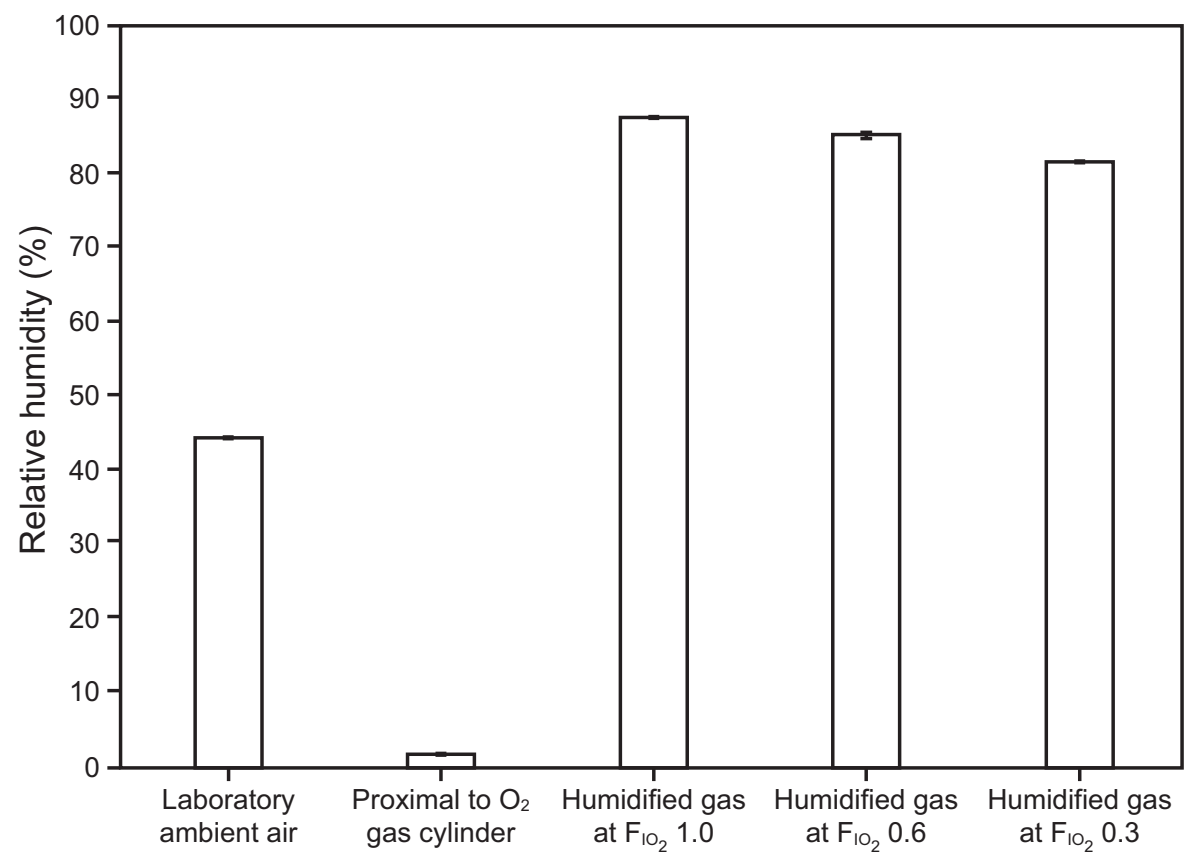

Fig. 7. The relative humidity at each condition. Each bar indicates the mean of either 1 min of observation or 20 breaths. The error bars denote SDs. The first 2 conditions are reference values to the latter 3 conditions, which are simulated breathing.

water reservoir. This method of pressure generation has been used for decades in other bubble CPAP systems. Bubbling in the water column superimposes oscillatory pressure waveforms on top of the CPAP level that may make bubble CPAP even more beneficial than other modes of CPAP delivery that do not generate pressure oscillations, for example, ventilator-driven CPAP. ${ }^{33}$ Even with these oscillations, the bubble CPAP system consistently and accurately delivered various mean CPAP levels throughout the study.
Preliminary data (see the supplementary materials at http://www.rcjournal.com) showed that the measured pressure was within $\pm 0.4 \mathrm{~cm} \mathrm{H}_{2} \mathrm{O}$ of the set pressure for a range of blended flows, which indicated that the resistance of the breathing circuit did not significantly affect delivered CPAP. Furthermore, with the lung simulator, pressures were observed to be within $\pm 0.5 \mathrm{~cm} \mathrm{H}_{2} \mathrm{O}$ of the set value regardless of the oxygen concentration being delivered. Therefore, pressure delivery was not impacted by the unique Venturi blender because the correct pressure was 


\section{Bubble CPAP for Low Resource Settings}

delivered regardless of the Venturi blender's setting. Overall, the bubble CPAP system delivered pressure well within $\pm 1.0 \mathrm{~cm} \mathrm{H}_{2} \mathrm{O}$, a metric consistent with bubble CPAP devices that are FDA cleared. ${ }^{34}$

Traditional Venturi blenders have not been used with infant CPAP systems before because they cannot generate any flow with back pressure as little as $0.6 \mathrm{~cm} \mathrm{H}_{2} \mathrm{O} .{ }^{35} \mathrm{~A}$ unique Venturi blender was recently described that can handle the low flows and thin tubing of infant oxygen delivery systems. ${ }^{29}$ This was the first published study to show highaccuracy oxygen delivery within a spontaneously breathing lung model when using a CPAP system that integrates an adjustable version of this unique Venturi blender. This represents a technologic breakthrough because other air-oxygen blending mechanisms in CPAP systems and other oxygen delivery devices for infants all require high-pressure air and oxygen inputs, electricity, compressors, oxygen concentrators, or expensive pneumatic blenders.

Because of these barriers, many providers in low- and middle-income countries currently give patients unblended $100 \%$ oxygen, which can cause retinopathy of prematurity, bronchopulmonary dysplasia, and neurologic injury. The WHO estimates that there are 1.4 million blind children worldwide, two thirds of whom live in nations where retinopathy of prematurity is a major contributor to blindness, which highlights the need for inexpensive and accurate oxygen delivery mechanisms. ${ }^{36} \mathrm{We}$ demonstrated consistent and accurate oxygen delivery across all CPAP and oxygen concentration levels in the bubble CPAP system for the tested model.

The WHO recommends starting CPAP at $30 \%$ oxygen and limiting use of higher oxygen concentrations. ${ }^{13}$ The bubble CPAP system successfully delivered oxygen concentrations from 30 to $100 \%$. The Venturi blender was most accurate at the lower settings, which are the most commonly used. For all oxygen concentration settings, the device delivered a blend of air and oxygen within 4 percentage points of full scale of the setting. This was comparable with the accuracy of commercially available pneumatic blenders ( $\pm 3 \%$ full scale) that are expensive, complex, and require pressurized air. ${ }^{37}$ Therefore, the bubble CPAP system delivered adequate range and accuracy of oxygen concentration settings in the tested model. Additional studies in vitro are needed to determine accuracy across all patient sizes by testing the system on larger infant and pediatric models.

The need for heated humidification is clear during mechanical ventilation, when the normal heating and humidifying functions of the nasopharynx are bypassed but is less clear during noninvasive ventilation. Some CPAP systems contain heated humidifiers, whereas others do not include any supplemental humidification but instead recommend applying nasal saline solution drops. Patients on these devices can experience nasal irritation and nose bleeds, which indicates that some humidification is appropriate..$^{38,39}$ The $\mathrm{WHO}^{40}$ and the American Association for Respiratory $\mathrm{Care}^{41}$ recommend humidification of oxygen delivered at flows greater than $4 \mathrm{~L} / \mathrm{min}$ to prevent drying of nasal mucosae. The question remains whether passive bubble humidification is adequate or if heated humidification is required during noninvasive ventilation. Many CPAP systems include an active heated humidifier, but these have drawbacks, including that they can cause condensation to accumulate in the circuit. Condensation may increase the infection risk and has been associated with nasal obstruction $^{42}$ and unintended increases in pressure levels. ${ }^{32}$

There are no defined standards for optimal temperature and humidity levels of CPAP breathing gases. Analysis of some data from adults suggests that the minimum absolute humidity is $15 \mathrm{mg} \mathrm{H} \mathrm{H}_{2} \mathrm{O} / \mathrm{L}^{43}$; whereas a heated humidifier commonly used to support neonates with bubble CPAP in high resource settings generates $23-27 \mathrm{mg} \mathrm{H}_{2} \mathrm{O} / \mathrm{L} .{ }^{42}$ The tested bubble CPAP system sources humidity both from the humidity present in entrained ambient air and from a passive bubble humidifier. Therefore, the amount of humidity generated by the device depends on the temperature and humidity of the environment. Based on preliminary data (see the supplementary materials at http://www.rcjournal.com), the bubble CPAP system generated 22.0-28.6 $\mathrm{mg} \mathrm{H}_{2} \mathrm{O} / \mathrm{L}$ in an environment at $30^{\circ} \mathrm{C}$ and $30 \% \mathrm{RH}$, which was comparable with the temperature and humidity levels of hospitals in India and Tanzania. The values of $22.0-28.6 \mathrm{mg} \mathrm{H}_{2} \mathrm{O} / \mathrm{L}$ were well over the minimum $15 \mathrm{mg} \mathrm{H}_{2} \mathrm{O} / \mathrm{L}$ and were comparable with commercial devices commonly used in high resource settings.

Furthermore, we observed $\mathrm{RH}>80 \%$ with the bubble humidifier in our cool, dry (44\% RH) laboratory. This level of humidity meets International Standardization Organization 8185:1997 - Humidifiers for medical use recommended values and is comparable with recently published data on noninvasive devices with active humidifiers applied to neonatal mannequins. ${ }^{44}$ This observation led to the assumption that exchange of humidity entrained through the Venturi blender (ambient) and humidifier combined with dried oxygen from the cylinder occurs very rapidly before entering the nasal cavity. Future studies should address whether additional increases in RH by bubble humidifiers are the result of increased water vapor, generation of aerosols, or both.

The bubble CPAP system was able to generate all tested flows with a 50 psi source of oxygen. Preliminary data (see the supplementary materials at http://www.rcjournal.com) showed that the blended flow of air and oxygen out of the Venturi was always greater than the flow of pure oxygen from the external source for oxygen concentrations $<100 \%$ due to the addition of entrained ambient air. This allows the user to conserve oxygen relative to improvised devices that source breathing gases only from pressurized oxygen sources. For example, consider an $\mathrm{M}$ tank filled to 2,200 psi 


\section{Bubble CPAP for Low Resource Settings}

$(7,080 \mathrm{~L})$ that will be refilled when it is $25 \%$ full. For a patient who needs $6 \mathrm{~L} / \mathrm{min}$ of breathing gases and $5 \mathrm{~cm} \mathrm{H}_{2} \mathrm{O}$ pressure on an improvised bubble CPAP system that provides $100 \%$ oxygen, the tank will last almost 15 hours. For the same patient on the subject bubble CPAP system at 30\% oxygen, the tank will last 26 hours.

Our study was limited in several regards. Only a preterm neonatal lung model was used, so further evaluation is needed to determine if the bubble CPAP system's reliable performance translates to larger infants. Furthermore, there is a limit to the extent that data from a mechanical lung simulator can be applied to actual patients. For example, the lung compliance and volume of patients on bubble CPAP may change over time, unlike in the lung simulator. Future studies in a dynamically breathing lung model may be useful to determine delivered pressure and oxygen over time. Our study did not consider oral leak and how a leaky seal around the nasal prongs may affect performance.

We also only used a set flow of $6 \mathrm{~L} / \mathrm{min}$ with the neonatal lung model. Understanding how higher flows affect performance would be worthwhile because precise control of flow with a meter may not be available in low- and middle-income countries. Also, bench-top studies such as this demonstrate efficacy of a device in ideal conditions, but in low resource settings where staff and biomedical engineering support are limited, it is difficult to create ideal conditions. Analysis of recent research has emphasized the challenges of implementing bubble CPAP treatment in low-resource settings. ${ }^{45}$ Further studies are needed to see how effective this novel system is when integrated into health-care facilities in different contexts.

\section{Conclusions}

To our knowledge this bubble CPAP system is the first infant CPAP device that is simple to use and provides CPAP with humidification and oxygen concentration control without the need for electricity, compressed air, or manual power. The results of this study demonstrated that the bubble CPAP system provided CPAP pressures, delivered oxygen concentration precision, and humidification comparable with commercial CPAP devices. The next steps are to build on the results of this study with further bench tests and to identify implementation strategies to integrate bubble CPAP systems effectively into health systems, optimize provider performance, and support quality care for newborns and infants in respiratory distress.

\section{REFERENCES}

1. World Health Organization. Children: improving survival and wellbeing. Available at: https://www.who.int/news-room/fact-sheets/ detail/children-reducing-mortality. Accessed Oct 27, 2020.

2. Singh GK, Yu SM. Infant mortality in the United States: trends, differentials, and projections 1950 through 2010. Am J Public Health 1995;85(7):957-964.
3. Heron M, Sutton PD, Xu J, Ventura SJ, Strobino DM, Guyer B. Guyer B Annual summary of vital statistics: 2007. Pediatrics 2010;125(1):415.

4. Lawn JE, Cousens S, Zupan J, Lancet Neonatal Survival Steering Team. 4 million neonatal deaths: when? Where? Why? Lancet 2005;365(9462):891-900.

5. Singhal N, Bhutta ZA. Newborn resuscitation in resource-limited settings. Semin Fetal Neonatal Med 2008;13(6):432-439.

6. Baraldi E, Filippone M. Chronic lung disease after premature birth. N Engl J Med 2007;357(19):1946-1955.

7. Aly H, Milner JD, Patel K, El-Mohandes AAE., El-Mohandes AA. Does the experience with the use of nasal continuous positive airway pressure improve over time in extremely low birth weight infants? Pediatrics 2004;114(3):697-702.

8. Jobe AH, Kramer BW, Moss TJ, Newnham JP, Ikegami M. Decreased indicators of lung injury with continuous positive expiratory pressure in preterm lambs. Pediatr Res 2002;52(3):387-392.

9. Polglase GR, Hillman NH, Ball MK, Kramer BW, Kallapur SG, Jobe AH, Pillow JJ. Lung and systemic inflammation in preterm lambs on continuous positive airway pressure or conventional ventilation. Pediatr Res 2009;65(1):67-71.

10. Thomson MA, Yoder BA, Winter VT, Martin H, Catland D, SilerKhodr TM, Coalson JJ. Treatment of immature baboons for 28 days with early nasal continuous positive airway pressure. Am J Respir Crit Care Med 2004;169(9):1054-1062.

11. Thomson MA, Yoder BA, Winter VT, Giavedoni L, Chang LY, Coalson JJ. Delayed extubation to nasal continuous positive airway pressure in the immature baboon model of bronchopulmonary dysplasia: lung clinical and pathological findings. Pediatrics 2006;118 (5):2038-2050.

12. Thukral A, Sankar MJ, Chandrasekaran A, Agarwal R, Paul VK. Efficacy and safety of CPAP in low- and middle-income countries. J Perinatol 2016;36 Suppl 1(Suppl 1):S21-S28.

13. World Health Organization. WHO recommendations on newborn health: guidelines approved by the WHO Guidelines Review Committee. Available at: https://apps.who.int/iris/handle/10665/ 259269. Accessed October 27, 2020.

14. Textbook of Neonatal Resuscitation (NRP). 7th ed. American Academy of Pediatrics and American Heart Association 2016, Gary M Weiner and Jeanette Zaichkin (editors)

15. Bomont RK, Cheema IU. Use of nasal continuous positive airway pressure during neonatal transfers. Arch Dis Child Fetal Neonatal Ed 2006;91(2):F85-F89.

16. Murray PG, Stewart MJ. Use of nasal continuous positive airway pressure during retrieval of neonates with acute respiratory distress. Pediatrics 2008;121(4):e754-e758.

17. Jani P, Luig M, Wall M, Berry A. Transport of very preterm infants with respiratory distress syndrome using nasal continuous positive airway pressure. J Neonatal-Perinatal Med 2014;7(3):165-172.

18. Dewez JE, Nangia S, Chellani H, White S, Mathai M, van den Broek N. Availability and use of continuous positive airway pressure (CPAP) for neonatal care in public health facilities in India: a cross-sectional cluster survey. BMJ Open 2020;10(2):e031128.

19. Baldursdottir S, Falk M, Donaldsson S, Jonsson B, Drevhammar T. Basic principles of neonatal bubble CPAP: effects on CPAP delivery and imposed work of breathing when altering the original design. Arch Dis Child Fetal Neonatal Ed 2020;105(5):550-554.

20. Heenan M, Rojas JD, Oden ZM, Richards-Kortum R. In vitro comparison of performance including imposed work of breathing of CPAP systems used in low-resource settings. PLoS One 2020;15(12): e0242590.

21. Brown J, Machen H, Kawaza K, Mwanza Z, Iniguez S, Lang H, et al. A high-value, low-cost bubble continuous positive airway pressure 


\section{Bubble CPAP for Low Resource Settings}

system for low-resource settings: technical assessment and initial case reports. PLoS One 2013;8(1):e53622.

22. Kumar MB, Deval N, Jangir NK. Technical assessment of a low cost multi-powered continuous positive airway pressure (CPAP) device. 2018 10th International Conference on Communication Systems \& Networks (COMSNETS), 2018/01/03/7, IEEE 2018.

23. Bennett DJ, Carroll RW, Kacmarek RM. Evaluation of a low-cost bubble CPAP system designed for resource-limited settings. Respir Care 2018;63(4):395-403.

24. John SC, Barnett JD, Habben ND, Le HT, Cheng E, John SP, Gustafson PA. Development and testing of a bubble bi-level positive airway pressure system. Respir Care 2017;62(9):1131-1136.

25. John SC, John AV, Moss AW, Gustafson PA, Fernando-Silva L, John SP. Bench testing of a bubble noninvasive ventilation device in an infant lung simulator. Respir Care 2020;65(9):1339-1345.

26. Myers S, Dinga P, Anderson M, Schubert C, Mlotha R, Phiri A, et al. Use of bubble continuous positive airway pressure (bCPAP) in the management of critically ill children in a Malawian paediatric unit: an observational study. BMJ Open Resp Res 2019;6(1):e000280.

27. Amadi HO, Okonkwo IR, Abioye IO, Abubakar AL, Olateju EK, Adesina $\mathrm{CT}$, et al. A new low-cost commercial bubble CPAP (bCPAP) machine compared with a traditional bCPAP device in Nigeria. Paediatr Int Child Health 2019;39(3):184-192.

28. Meara JG, Leather AJM, Hagander L, Alkire BC, Alonso N, Ameh EA, et al. Global Surgery 2030: evidence and solutions for achieving health, welfare, and economic development. Lancet 2015;386 (9993):569-624.

29. Mollazadeh-Moghaddam K, Burke TF, Dundek M, Yeung SH, Sharma R, Ravi R, et al. A low-cost Venturi ambient air-oxygen blender for neonatal oxygen therapy. Acad J Pediatr Neonatol 2020;8 (5):48-56.

30. DiBlasi RM. Nasal continuous positive airway pressure (CPAP) for the respiratory care of the newborn infant. Respir Care 2009;54 (9):1209-1235.

31. Polgar G, Kong GP. The nasal resistance of newborn infants. J Pediatr 1965;67(4):557-567.

32. Youngquist TM, Richardson CP, DiBlasi RM. Effects of condensate in the exhalation limb of neonatal circuits on airway pressure during bubble CPAP. Respir Care 2013;58(11):1840-1846.

33. Poli JA, Richardson CP, DiBlasi RM. Volume oscillations delivered to a lung model using 4 different bubble CPAP systems. Respir Care 2015;60(3):371-381.
34. U.S. Food and Drug Administration, Department of Health \& Human Services. Seattle-PAP Noncontinuous ventilator (IPPB) 510(k) PMA 21 CFR 868.5905 approval letter, October 11, 2013. Retrieved May 28, 2021, Available https://www.accessdata.fda.gov/cdrh_docs/pdf13/ K131502.pdf.

35. Jones JG. The 'fixed performance' venturi: effect of downstream pressure on outflow and $\mathrm{FiO}_{2}$. Anaesthesia 2004;59(7):704-709.

36. World Health Organization (2000) Preventing blindness in children. Report of a WHO/IAPB Scientific meeting. WHO/PBL/00.77 Geneva: WHO; 2000.

37. U.S. Food and Drug Administration, Department of Health \& Human Services. Precision Medical, Inc Blender 510(k) PMA 868.5330 approval letter, January 10, 2006. Retrieved May 28, 2021, Available from https://www.accessdata.fda.gov/cdrh_docs/pdf5/K053232.pdf. Accessed October 27, 2020.

38. Mwatha AB, Mahande M, Olomi R, John B, Philemon R. Treatment outcomes of Pumani bubble-CPAP versus oxygen therapy among preterm babies presenting with respiratory distress at a tertiary hospital in Tanzania—randomised trial. PLoS One 2020;15(6):e0235031.

39. Kawaza K, Machen HE, Brown J, Mwanza Z, Iniguez S, Gest A, et al. Efficacy of a low-cost bubble CPAP system in treatment of respiratory distress in a neonatal ward in Malawi. PLoS One 2014;9(1):e86327.

40. World Health Organization. Oxygen therapy for children: a manual for health workers. Available at: https://www.who.int/maternal_child_ adolescent/documents/child-oxygen-therapy/en/. Accessed October 27, 2020

41. Kallstrom TJ, American Association for Respiratory Care (AARC). AARC Clinical Practice Guideline: oxygen therapy for adults in the acute care facility -2002 revision \& update. Respir Care 2002;47 (6):717-720.

42. Flink RC, van Kaam AH, de Jongh FH. A humidifier in the invasive mode during noninvasive respiratory support could increase condensation and thereby impair airway patency. Acta Paediatr 2018;107 (11):1888-1892.

43. Lellouche F, Maggiore SM, Lyazidi I, Deye N, Taillé S, Brochard L. Water content of delivered gases during non-invasive ventilation in healthy subjects. Intensive Care Med 2009;35(6):987-995.

44. Roberts CT, Kortekaas R, Dawson JA, Manley BJ, Owen LS, Davis PG. The effects of non-invasive respiratory support on oropharyngeal temperature and humidity: a neonatal manikin study. Arch Dis Child Fetal Neonatal Ed 2016;101(3):F248-F252.

45. Wilson PT. Bubble CPAP in resource-poor settings: friend or foe? Lancet Respir Med 2019;7(11):923-925. 